\title{
An Environment for Object-Oriented Real-Time Systems Design
}

\author{
Rob van de Weg, Rolf Engmann \\ Computer Science Department, University of Twente, P.O. Box 217, \\ NL-7500 AE Enschede, The Netherlands, E-mail: \{vandeweg I engmann\}@cs.utwente.nl \\ Raoul van de Hoef \\ Moret Ernst \& Young, P.O. Box 3101, NL-3502 GC Utrecht, The Netherlands, \\ E-mail: rhoef@mc.mey.nl \\ Vincent ten Thij \\ Moekotte Enschede bv, P.O. Box 666, NL-7500 AR Enschede, The Netherlands
}

\begin{abstract}
A concise object-oriented method for the development of real-time systems has been composed. Hardware components are modelled by (software) base objects; base objects are controlled by a hierarchy of coordinator objects, expressed in an organizational diagram. The behaviour of objects is specified by state transition diagrams. This approach considerably promotes requirements analysis and communication with the customer. A CASE tool has been constructed with diagram editors for graphical specifications of real-time systems. The tool can generate executable code for PLCs from these graphical specifications; reuse of previous results is supported by the repository function of the tool. Experiences attained in practice with method and tool show that time spent in system testing and installation is reduced considerably.
\end{abstract}

\section{Introduction}

In the past several methods for the analysis and design of technical real-time systems have been published, see e.g. [1 - 8]. However, according to our experiences these methods do not always meet the requirements of small companies having their business in designing and constructing control systems, e.g. for manufacturing and chemical processes. In many cases these methods offer specification techniques that are redundant or require too much detail, or they are too rigorous in their procedures. This is caused by the fact that most of the required functionality and hardware objects are already captured by socalled process \& instrumentation diagrams (P\&IDs). A P\&ID is the result of the earlier hardware engineering process. The objective of real-time system development is the design of the software control process of the hardware objects.
Two main issues in control systems that we have studied are:

- coordination and cooperation of hardware components;

- failures of hardware components.

According to our experiences there is not enough attention for these problems in existing methods.

In this study we adopt the following definition of a realtime system: a real-time system is any information processing activity or system, which has to respond to externally generated input stimuli within a finite and specified period [9]. Real-time systems are characterized by parallel processes and operations, coordination of actions of many rather independent components, detection and handling of failures, interrupts, time-outs and conflicting goals.

Many companies involved in the development of realtime systems meet the following problems:

- there is no uniform development method available, designers use individual, informal procedures;

- absence of explicit standards for specifications of product design, which hampers communication between designers and customers;

- insufficient checks on the completeness, consistency and correctness of design specifications and documentation;

- limited or insufficient systematic procedures for testing and simulation.

The main goal of the present research was to compose a practical method for the development of real-time systems in which detecting and handling failures are completely embedded. In addition to this main objective we have the following subgoals with respect to the method:

- support for a company's ISO-9001 certification;

- increased effectivity and efficiency compared with the conventional design process;

- reduction of complexity in the design of real-time 
systems;

- uniform and unambiguous procedures;

- specifications should easily be verified;

- reuse of specifications and documentation for the procurement and design processes;

- user-friendly CASE tool support for generating specifications (diagram editors);

- automatic generation of code for several types of PLCs (Programmable Logic Controllers) and microcomputers.

As a result the method CORSO was developed; the acronym CORSO is derived from a Dutch phrase for "CASE tool support for real-time systems design". It should be admitted that the CORSO method may not be applicable to all kinds of real-time systems design. Therefore, the relevance of the method for solving specific real-time problems is a research topic.

The steps in method engineering that we have followed in this project are:

- composition of the CORSO method;

- construction of a CASE tool supporting the method;

- realization of the code generator.

A basic concept in the method is coordination [10]. Coordination encompasses scheduling and control of different independent, unrelated objects. Coordination represents a level of abstraction above the conceptual level of individual objects and permanent relationships in the well-known ANSI/SPARC architecture. Coordination is discussed in more detail in section 2.1 in the context of the organizational model.

Presently, methods and corresponding commercial tools are available, e.g. OOA [6], OMT [4], ROOM [5], Statecharts [11]. However, in these methods hierarchical control structure and error handling are less pronounced. The CORSO method and tool support the issues of coordination, fault tolerance and code generation explicitly.

The CORSO method is described in chapter 2; the models and diagrams are explained with examples and method-data models. Chapter 3 contains a short discussion on possible failures that may occur in technical systems. The CASE tool and code generation supporting the CORSO method are described in chapter 4 . Chapter 5 contains conclusions and some research questions.

\section{The CORSO method}

The CORSO method intends to promote a structured and efficient development of control software for realtime, technical systems. The method is based on the object-oriented paradigm, in which software objects are responsible for the correctness of their own operations and data $[1,4,6]$. One of the first activities in the method is to identify the relevant entities (hardware components) that should be modelled by (software) objects, so-called base objects in the real-time system.

The analysis of the system may start from a technical specification of the hardware. In many cases such a specification is represented in a technical layout, for example a Process and Instrumentation Diagram (P\&ID), showing which hardware components are to be controlled and which sensors are available to collect data on the operation of the hardware system. Such a diagram may be helpful in the identification of the relevant hardware components and sensors.

The initial activities of the method are the identification of the following items in the technical system to be controlled:

- hardware components (actuators) with their relevant commands, for example valve: open, close;

- sensors with the meaning of their signal, for example elevator door sensor: door is open;

- external events detected by sensors, for example temperature exceeds safety limit.

The list of hardware components is the starting point for the design of the control structure, which is specified in the so-called organizational model. The organizational model represents the structural cooperation between software objects. The actual behaviour of each object (the life history or life cycle, see e.g. [6]), is specified in a so-called behavioural model. These modelling techniques are complementary to the P\&ID technique. The selection of the modelling techniques is based on a unifying object-oriented framework [10], in which all concepts to be captured in the systems analysis are identified.

\subsection{The organizational model}

The organizational model specifies in detail how software objects in the control system will cooperate. The organizational model is expressed in a diagram showing a hierarchical structure; figure 1 shows a simple example of such a diagram, as it has been realized in the CASE tool supporting the CORSO method, described in chapter 4. The nodes of the diagram represent software objects; yokes represent the control structure between the objects. The nodes on the lowest level are the so-called base $o b$ jects, which directly control corresponding hardware components.

Base objects model the behaviour of hardware components. In this context the relevant hardware components can independently receive and handle command signals and usually they can transmit their status to the control system via feedback signals. A base object controls just one specific hardware component; the base object initiates operations to be executed by the corresponding hardware component by sending commands, and monitors via feed- 


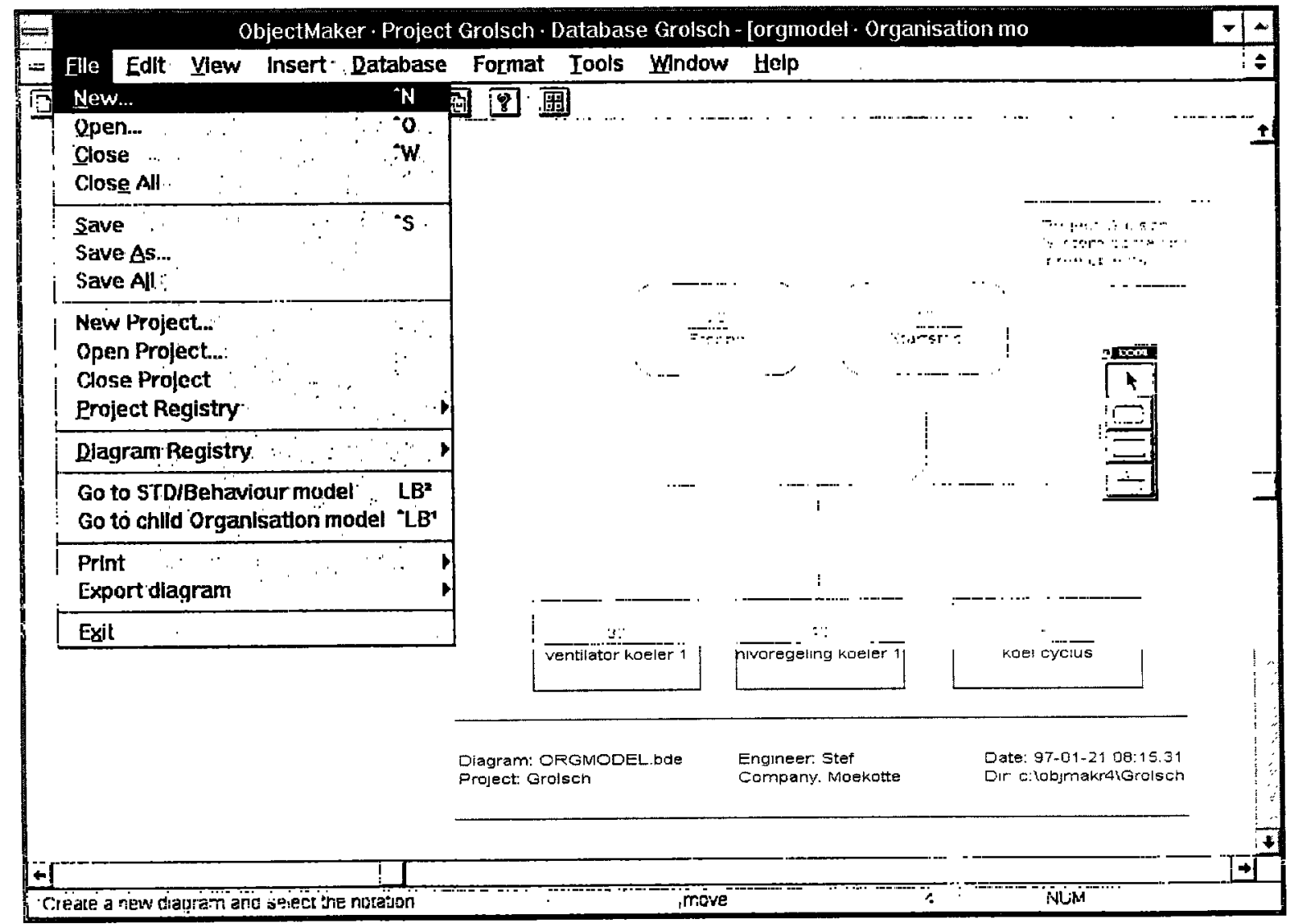

Figure 1. Corso tool screen with an organizational diagram

back signals received from sensors whether the desired operation is carried out correctly and timely.

The actions of base objects are coordinated and controlled by a hierarchy of supervisory objects, the so-called $\mathrm{co}$ ordinator objects or coordinators. Coordinators trigger the execution of operations of base objects (or other coordinators); in turn, base objects trigger the execution of operations of hardware components. Coordinator objects are introduced in the design of a control system when actions of subordinated objects (either base objects or coordinator objects) are executed in parallel or should be scheduled due to limited resources. Introduction of coordinators may also decrease the span of control of objects and in this way increase the surveyability of complex systems for purposes of design, realization, testing, and maintenance.

If the operation of a hardware component fails, the corresponding base object may take corrective action, for example repeat the command. If a corrective action doesn't succeed (for example in case of an irrecoverable failure) the situation is a so-called exception; the base object communicates details on the exception to the coordinator that triggered the action. The coordinator may try to reallocate the responsibility for execution of the action to another base object; if this is not possible the coordinator may terminate the activities of the base object(s) involved and of the corresponding hardware components. Further, it may notify its superior coordinator or the user or operator of the system the detection of an exception. Alternatively, control may be passed to a superior coordinator.

The organizational model is hierarchical in the sense that each object controls one or more subordinated objects. However, each object may be controlled by one or more coordinators, so the organizational model has not necessarily a tree structure. The meta-data model of the organizational diagram is shown in figure 2.The entityrelationship (ER) diagram notation is adopted from [12] with structural constraints expressing participation of entity types in relationship types. An additional constraint, not expressed in the ER-diagram, is that a coordinator object always controls at least one other (base or coordinator) object.

The organizational model as it is described here corresponds to a large extend with the object communication model (OCM) in the Object-Oriented Analysis (OOA) method described by Shlaer \& Mellor [6]. 


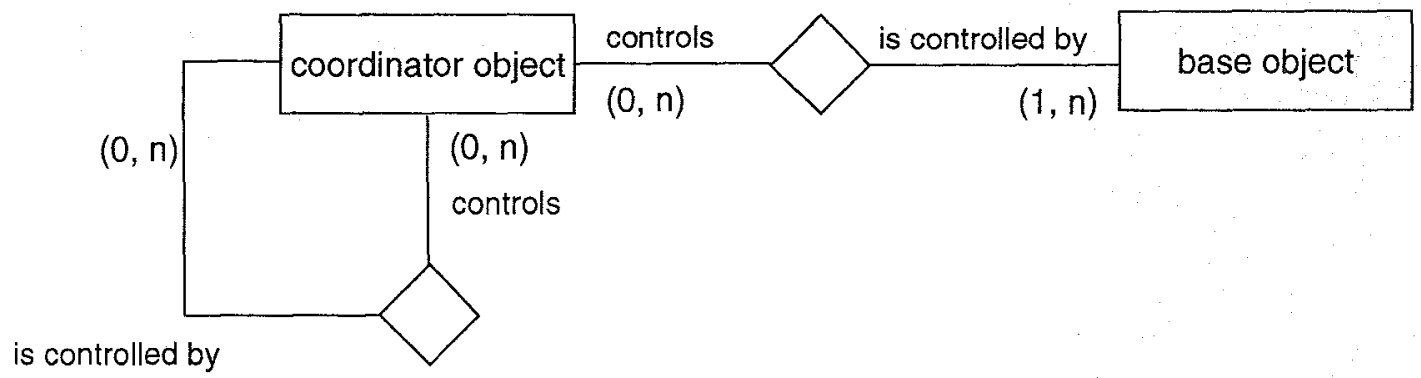

Figure 2. Method-data model of the organizational diagram

\subsection{The behavioural model}

The hardware and software objects may be conceived as state machines, whose temporal behaviour can be specified by means of state transition diagrams. The type of state transition diagram (STD) used in the behavioural model is the variant corresponding with Moore state machines (See e.g. [13], p. 374.) Actions performed by an object are associated with its states, as an action performed by a hardware component may take a significant amount of time, and may fail due to hardware failures. Fault tolerant modelling can be supported effectively by guarding the proper and timely termination of actions. The choice for Moore machines is also a pragmatic one; it is rather straightforward to translate them to PLC code.

Actions in coordinator states may be interpreted by subordinated objects as conditions that trigger a state transition; other actions may induce operations on timers, counters, registers, Boolean variables, and functions, or generate output signals to hardware components (actuators).

2.2.1. States and state transitions. In the behavioural model we distinguish four types of states: active states, passive states, failure states, and initial states. The notation shown in this paper corresponds with the behavioural diagrams as realized in the CASE tool. States are represented by a circle containing the name of the state and the ac- tion(s) performed by the object in that state.

Passive state. In a passive state the object is in a stable situation, which may last for an indefinite period, or for a period that in general can not be predicted. Examples are the open position of a valve in a water conduct, the movement of a vehicle or an elevator, the operation of a production unit in a continuous process.

In general, a state is of the passive type, if the transition to another state is caused by an internal command signal (action) from a (superior) coordinator, or an external event (external command signal), or detection of a hardware failure. A passive state is represented by a circle with a normal circumference.

Active state. In an active state the object is temporarily in a transient situation that by its nature should terminate within a predictable period. Examples are opening a valve in a pipe line, closing the door of an elevator, filling a tank with fluid up to a specific level.

In general, a state is of the active type, if the transition to another state is caused by a subordinate object or hardware component reaching a specific state, or by a feedback signal from a sensor. An active state is represented by a circle with a broken circumference.

Failure state. An object may enter a failure state caused by a failure occurring in the controlled (hardware) system.

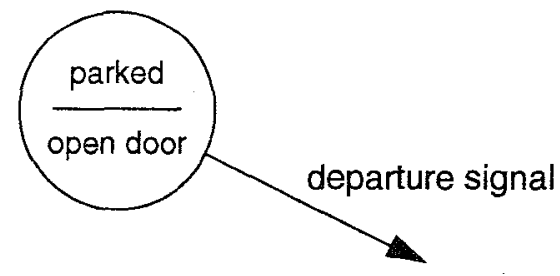

Figure 3. Passive state 'parked' with action 'open door' and transition with condition 'departure signal' 


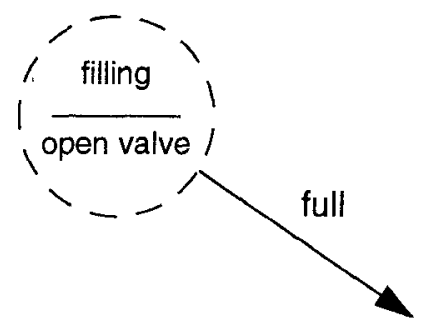

Figure 4. Active state with transition

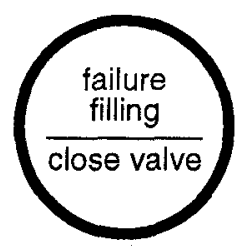

Figure 5. Failure state

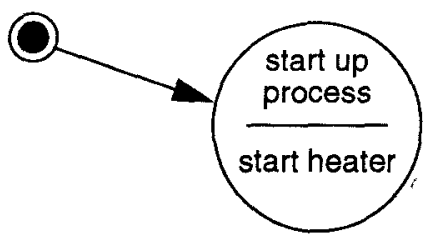

Figure 6. Initial state and corresponding first state

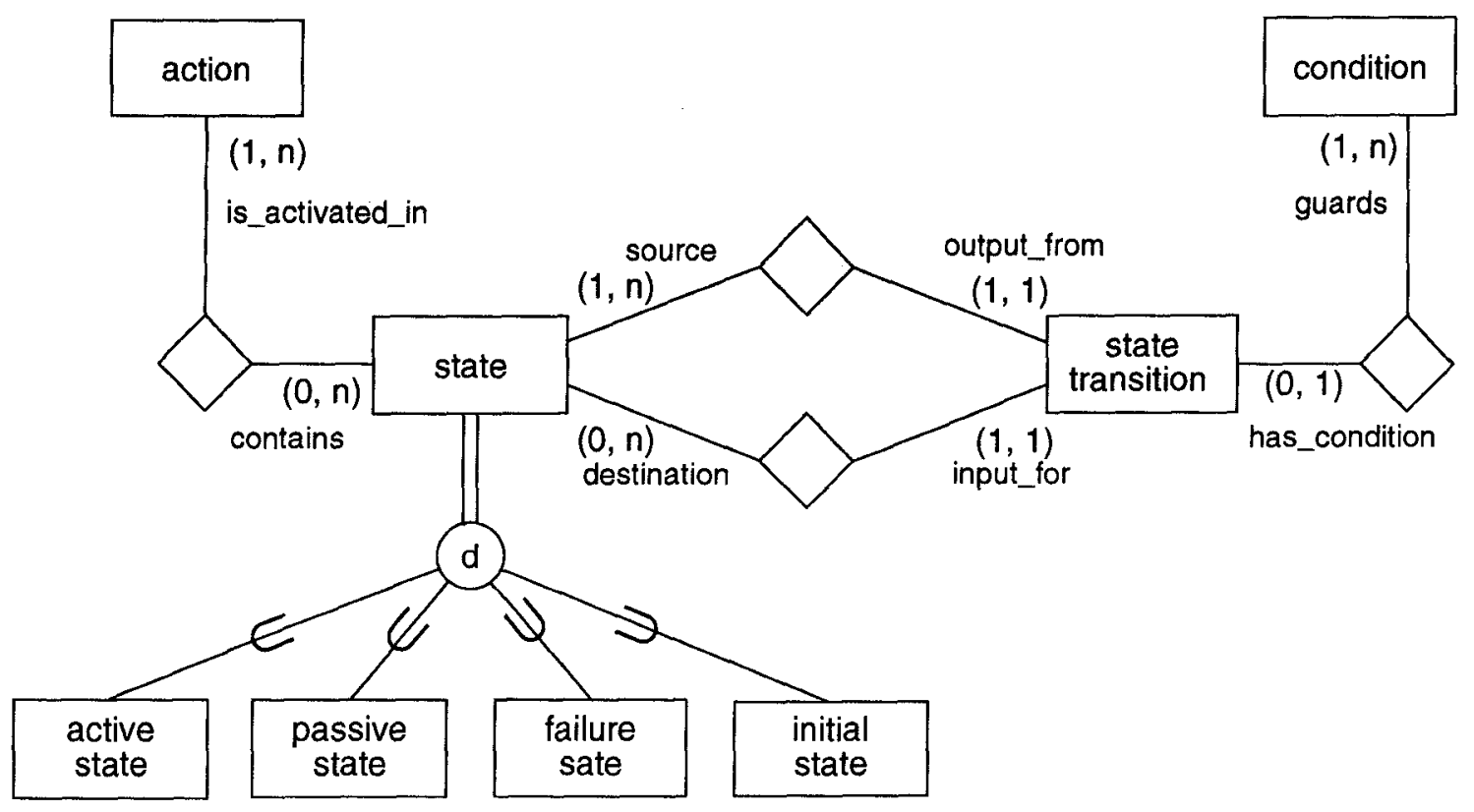

Figure 7. Method-data model of the behavioural diagram 
A failure state of an object can be detected by a superior coordinator, which may take appropriate action. In general, an object may leave a failure state due to a command signal. A failure state is represented by a circle with a bold circumference.

Initial state. The initial state is a virtual state denoting the entry point of the behavioural model when the system is energized. It is represented by a small circle containing a bullet and an unconditional transition to a passive or active state, representing the first (real) state in the behavioural model entered by the object. Each behavioural model should have one and only one initial state.

State transition. State transitions are represented by arrows, annotated by a Boolean condition. Such a condition may be complex, i.e. consisting of simple conditions connected by logical operators (AND, OR, NOT). Control signals and command signals can be used as simple conditions.

Other possible simple conditions are input signals (sensor signals from the hardware), Boolean values, comparisons of values, the state of timers, the completion of the execution of functions. When an object is in a specific state and the condition of a transition from that state is true then the transition is enabled, and the object state is changed from the source state to the destination state of that transition. The method-data model of the behavioural diagram is shown in figure 7.

A condition on a state transition may contain commands from a superior coordinator; in this case such a command appears as an action of that coordinator. In a coordinator object a condition on a state transition may contain the name of a state of a subordinated object, which is interpreted as a feedback signal. An example of a behavioural diagram is shown in figure 8 .

2.2.2. Special constructs used in behavioural models. Additional constructs are available for controlling state transitions in behavioural models: timers, counters, registers, Boolean values, general functions.

Timer. Timers are used to check the duration of operations that should terminate within a specific time period. Timers are frequently used in combination with active states, for example to check whether a valve is closed in time. In

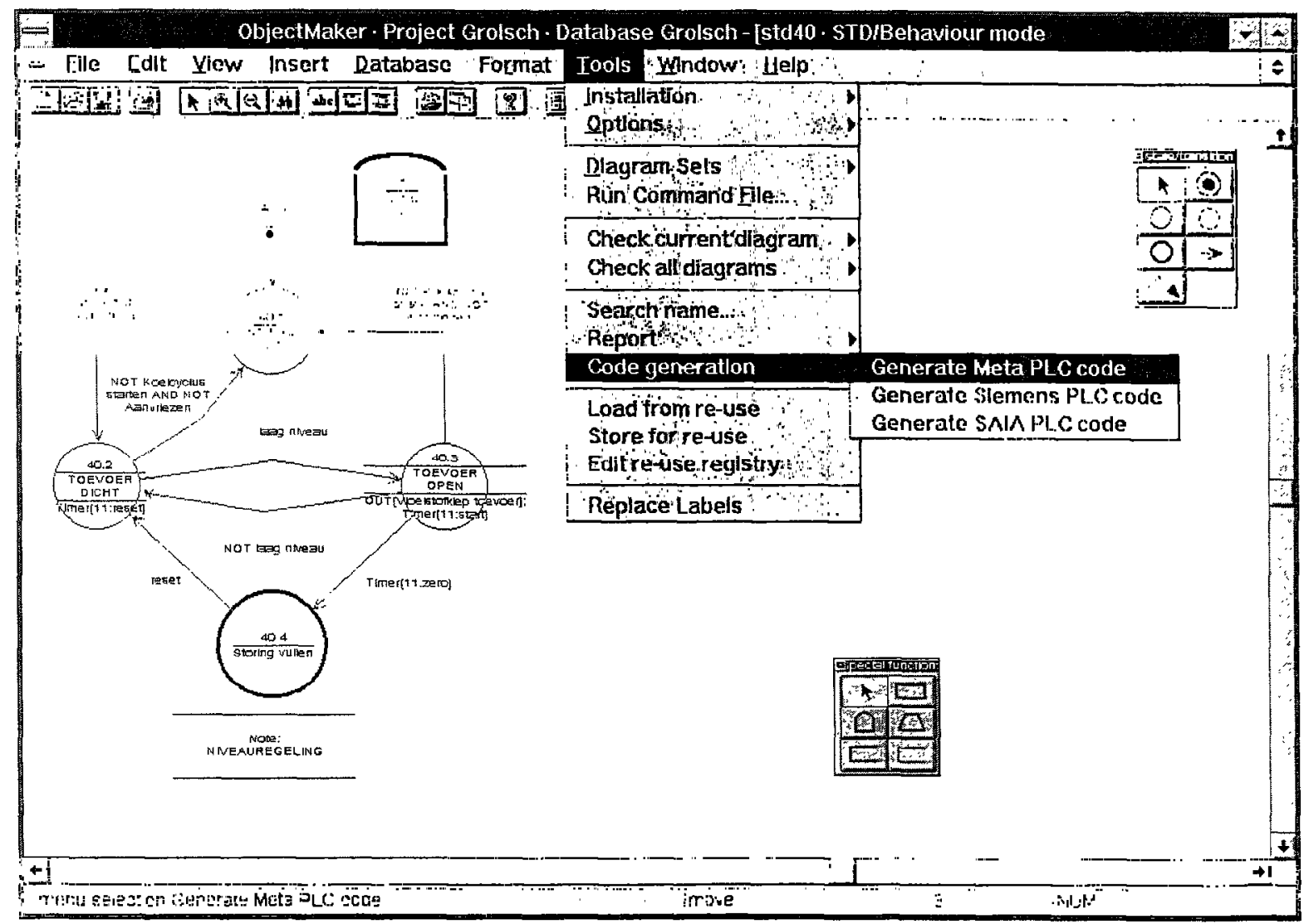

Figure 8. Corso tool screen with a behavioural diagram 
case of a time-out a failure may have occurred, appropriate action should be taken, and a warning message may be sent to the operator.

Counter. Counters can be used to count events. A counter can be set to a specific value and reset to 0 , and incremented and decremented by 1 . The test whether a counter has the value zero may be used as a simple condition on a state transition.

Register. Registers (or data stores) are used to store values. The values stored in registers can be compared with the operators EQ, NEQ, LT, LE, GT, GE. For example a condition on a transition could contain the simple condition GT[RA, RB], which tests if the contents of register $\mathrm{RA}$ is greater than the contents of register $\mathrm{RB}$.

Boolean variable. A Boolean variable (or data bit) is used to store a condition. A Boolean variable can be set to the values true and false. The name of the Boolean variable can be used as a simple condition.

Function. Functions are used to express more complex actions in states that can not be represented graphically, e.g. numerical calculations. Functions are strictly local for objects and do not affect other objects. In the code generation step a function should manually be filled in with the appropriate PLC code. Functions are integrated into the behavioural model.
2.2.3. Input/output signals. The input and output signals that are exchanged between objects and environment (sensors and actuators) are specified in the so-called input/output list. The control system receives input signals from sensors, and generates output signals to control the hardware. In the input/output list each signal is described by its name and a number, indicating its bit address.

The receipt of an input signal from a sensor is an event, which may be interpreted as a condition enabling a state transition in an object. A state of an object may contain an action generating an output signal to an actuator. An example of an action sending an output signal is OUT[start motor], in which 'start motor' is the name of an output signal.

Input signals can be distinguished into momentary or transient signals, and continuous signals. Transient signals may be electronic pulses corresponding to the occurrence of an event, for example a passenger pressing an elevator summons button.

Continuous signals may reflect the current state of a hardware component, for example the signal from the 'door closed' sensor of an elevator door. Such a signal can be used to check the correspondence between the environment and the state of a software base object.

2.2.4. Internal signals. Mutual communication among objects is realized by means of internal signals, which are exchanged between a coordinator and its subordinate objects. In the behavioural model we distinguish command

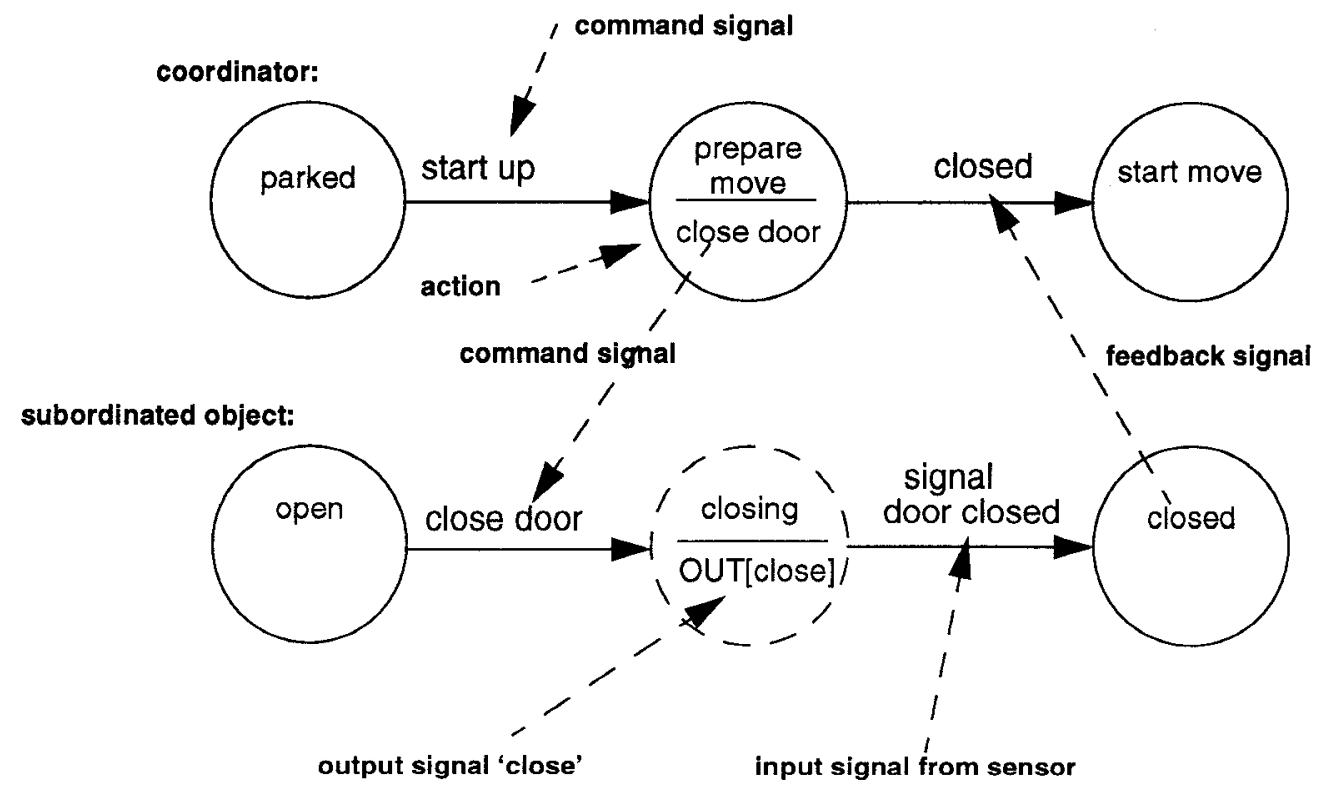

Figure 9. Command signals and control signals 
signals and feedback signals.

A command signal is sent by a coordinator to one or more subordinate objects. A command signal should be specified as an action in one of the states in the behavioural model of the coordinator. The name of the action should be used in a condition on a state transaction of the subordinate object receiving the corresponding command signal. So, a command signal is always sent in a downward direction with respect to the hierarchy, expressed in the organizational model.

A feedback signal is sent by a subordinate object to its (superior) coordinator; it should be specified as the name of a state in the behavioural model of the subordinate object. The name of the state is used in a condition on a state transition of the coordinator receiving the control signal. So, a feedback signal is always sent in an upward direction with respect to the hierarchy, expressed in the organizational model. A schematic summary on signals is shown in figure 9.

Signals may not be exchanged between objects on the same level. Communication between objects on the same level should only be realized via a coordinator on a higher level, otherwise the hierarchical control structure would be violated.

2.2.5. Rules for behavioural models. Behavioural models should be checked for correctness and completeness. Many syntactical checks are supported by the CORSO CASE tool, described in the following. The designer should take care of the semantic correctness of the models. Some semantic rules for behavioural models are:

- passive states may only be connected directly by a state transition if the transition is momentary and cannot be prevented or hampered by failing components in any way, otherwise there should be at least one active state between two passive states.

- an action corresponding to an active state needs time and may not be completed in time or not terminate at all due to failures. Therefore an active state should be guarded by a timer; in case of a time-out an exception has occurred, which may cause a transition to a failure state.

- a state corresponding to a detected continuous external signal may have a state transition to a failure state if the signal drops without specific reason. In such a case there might be an inconsistency between the control system and the hardware. An example of inconsistency is given in the next chapter.

\section{Failure analysis}

To promote resilience and safe operation of a system it is necessary to analyse the possible failures in the hard- ware, and develop mechanisms to detect and handle them. Base objects should be designed in such a way that they can be reused in many projects. For failure handling this means that:

- failures should be handled and solved locally if possible. Otherwise a failure should be reported to a superior coordinator on the next higher level;

- failures may not be transferred to objects at the same level.

In base objects the following exceptions may occur.

- Time-out: this type of failure should always be considered in active states. An active state may take too much time due to failing components. In case of a time-out a state transition to a failure state should occur.

- Inconsistency: an inconsistency between the software and the hardware may be detected by continuous input signals. An example is the absence of the input signal 'door closed', implying that an elevator door is not closed or the sensor is defective. If the corresponding software object is in the state 'closed', then there is a discrepancy, which cannot be solved and may cause a dangerous situation. In this case a transition to a failure state should follow.

- Hardware failure: a hardware component itself may indicate a failure, for example a motor that is defective. Detection by the software implies a corresponding input signal emitted by the defective component.

In coordinators two types of failures may be detected.

- Inconsistency: specific combinations of states of subordinate objects of a coordinator may be forbidden or impossible. An example is an elevator in the state 'moving', and the elevator door in the state 'door open'; in such a case the coordinator should detect an inconsistency corresponding to a potentially dangerous situation, and take corrective action, e.g. halt the elevator, ring alarm.

- Failure in a subordinate object: in general, failures should be handled locally by a base object, but in case of irrecoverable errors that cannot be repaired independently, an object may report an exception to a superior coordinator.

If a failure may occur then a failure state has to be added to the corresponding behavioural model. For each failure state there should be a state transition to a normal (passive or active) state. There are several standard transitions from a failure state:

- transition to the previous state, before the failure occurred;

- transition to the previous active state; the object should pass from that active state to the correct passive state; 
- transition to the initial state; in this case the system executes a restart operation.

Issues on failure analysis and fault tolerance are discussed in the literature $[14,15]$.

\section{Architecture of the CORSO environment}

\subsection{CASE tool}

To support the CORSO method a CASE tool has been realized. The CORSO tool was constructed based on the ObjectMaker meta-CASE tool [16]. The tool contains diagram editors for drawing organizational and behavioural diagrams as has been specified in chapter 2 . The methoddata models of the diagrams are shown in figures 2 and 7 . Diagrams are stored in the repository of the tool.

A new system development project is normally started by drawing an organizational diagram. By clicking the mouse button on an object in the organizational diagram control is passed to the behavioural diagram editor to create or retrieve the corresponding behavioural diagram. The tool is menu driven; existing diagrams can be retrieved from the repository, changed, printed, and stored.

Checks on consistency and completeness. The CORSO tool can perform checks on the consistency and completeness of the diagrams specified in a project. These checks can be applied to individual diagrams as well as to the complete set of diagrams in a project.

An example of a check on a behavioural diagram is that each state transition should have a condition. A check on the consistency between different behavioural diagrams is that a signal used in a condition should be defined either as an action (command signal), as a state (feedback signal), or as an input signal (in the input/output list). An example of a check pertaining to an organizational diagram and the corresponding behavioural diagrams is that for each object in the organizational diagram a behavioural diagram should exists.

The user can initiate a check operation on the current diagram or on all diagrams of the current project at any moment. The tool will then produce a report showing the errors detected in the diagram or diagrams. Another service of the tool is the generation of listings of signals and states in the behavioural models.

Code generation. The behavioural models are used for the generation of code in two steps. In the first step the STDs are translated to an intermediate textual code. This intermediate code is PLC independent. In the second step the intermediate code is converted to specific PLC code. Presently, compilers to convert intermediate code to PLC code for Siemens and SAIA PLCs are available in the CORSO tool. Figure 10 gives a schematic summary of the architecture of the CORSO tool.

Reusable specifications. In practice real-time systems are composed from a limited number of technical components, such as valves, pumps and motors. It is not necessary to specify behavioural models for such standard

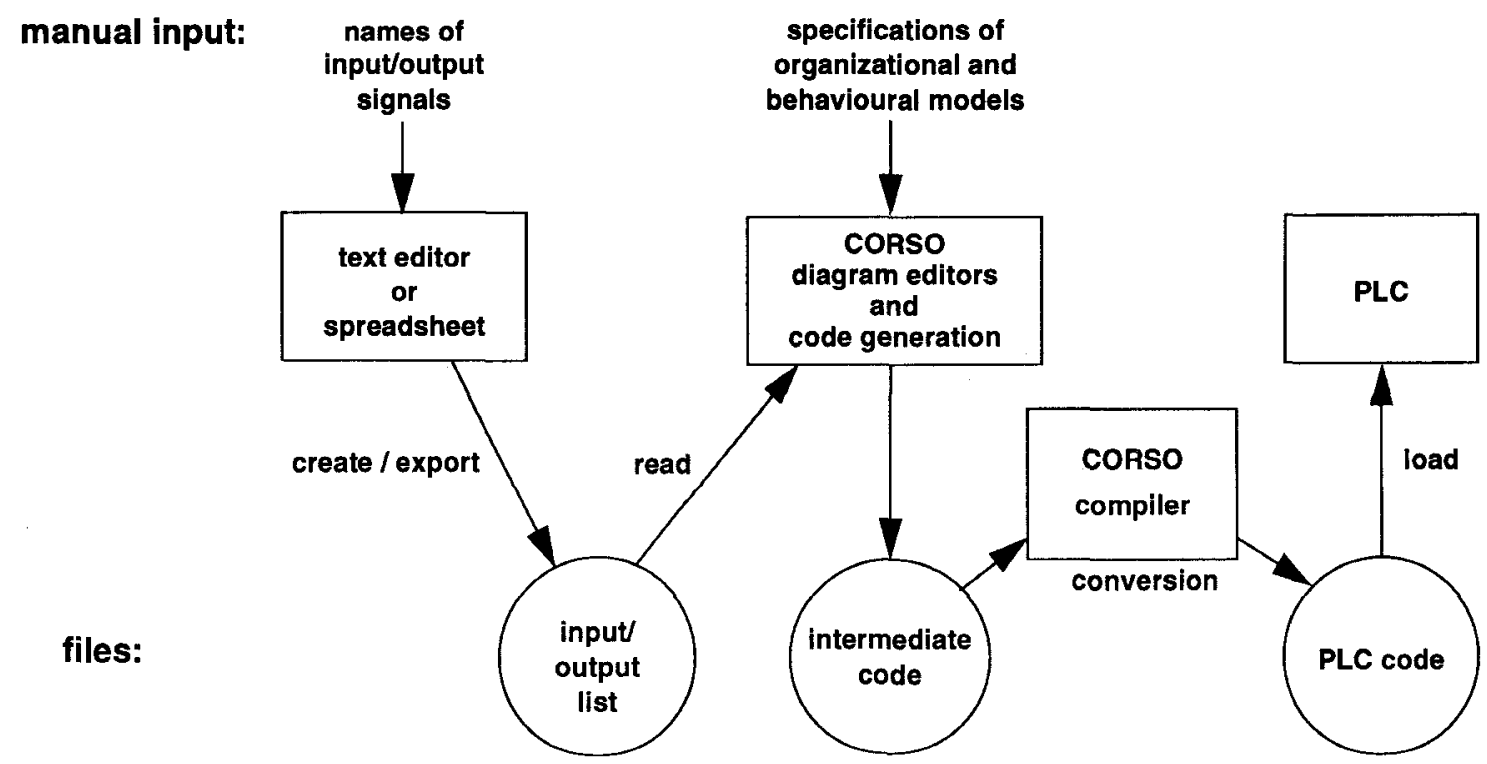

Figure 10. CORSO tool architecture 
components for each project. Behavioural models can be specified once and stored for reuse in the CORSO repository. In a specific project behavioural models can be retrieved from the repository and inserted in the current project. In this way the efficiency and reliability of the design process is increased considerably.

\subsection{The CORSO method and CASE tool support}

The CORSO method contains the following steps.

Technical analysis. Development starts from a technical specification of the hardware system to be controlled. Such a specification may be expressed in technical drafts or diagrams, for example a Process \& Instrumentation Diagram (P\&ID) and textual descriptions of the functions performed by the system.

From the technical specifications the individual hardware components to be controlled and monitored should be identified. Also information on sensors and input and output signals should be collected. Incoming signals may correspond with events to which the system may have to react; therefore possible events should be listed with their signals and the sensors that receive these signals.

Analyse possible failures that may occur in the system. Specify how failures may be detected and how they should be handled by the control system.

Identify objects and coordinators, specify organizational model. Identify the hardware components that should be modelled by software objects in the control system. These software objects are the base objects, which appear as the leave nodes in the organizational model.

Specify coordinators for base objects that perform actions simultaneously or that need a common control mechanism. In the same way specify coordinators at a higher level for coordinating subordinated coordinators. In this way the organizational model is developed until the root coordinator at the top level is specified. Identify command and feedback signals exchanged by objects on different layers of the organizational model.

The organizational model is entered into the CASE tool via the corresponding diagram editor. External signals should be specified in an input/output list.

Specify behaviour. For each object/coordinator specify a behavioural model with the behavioural diagram editor. Identify states, transitions and conditions on transitions. Existing behavioural models from previous projects, stored in the repository may be used in the present project. Add special functions if necessary.

Checks. Run consistency and completeness checks by the tool, and correct the diagrams if necessary.
Code generation. If no errors are detected first intermediate code can be generated. Next, PLC code for a specific type of PLC can be obtained by converting the intermediate code.

\section{Conclusions and future research}

The CORSO method has been proven to be an effective aid in developing several production and control systems. A recent commercial project in which CORSO was used is the development of a chilling-room for a brewery. The diagrams shown in figures 1 and 8 are taken from this project. From a practical point of view the CORSO approach has the following characteristics:

- Software is developed in a very structured and formalized way; in requirements analysis problems, incompleteness and inconsistency are detected and can be discussed with the customer in a very early stage.

- Time needed for development, acceptance tests and installation has been decreased considerably due to increased reliability and less design errors. Productivity in using the CORSO method and CASE tool in projects has increased by a factor of about 2.5.

- Reuse of design improves the results of project proposals and systems analysis.

- The attention of the developer is focussed on analysis and design rather than on coding. Changes need only be made in the organization or behavioural diagrams. Therefore, the software development process is shorter and the quality of the results is improved.

- Our approach in creating an environment for systems development can also be applied to other companies and situations, e.g. with different traditions in diagram techniques.

Present and future research is directed towards the application field of the method, simulation facilities, debugging, and failure analysis.

Application field. The number of projects and experiences with the CORSO method is modest. It should be investigated for which types of real-time systems and in which environments the method and the tool are especially suitable.

Simulation. Present research is directed towards simulation facilities for testing the behavioural models. While simulating, the behavioural diagrams are shown on a windows screen; input signals can be activated by screen buttons, output signals are shown by screen indicators. The behaviour of an individual object is visualized by showing its behavioural model, the present state and output signals. 
Debugging facilities. It is expected that the method and the tool will be effective in maintenance if a trace of the states and transitions of the objects is logged and stored in a data base, at least for a limited time. In case of a failure it should be possible to analyse the recent behaviour of the objects, which may support trouble shooting in an effective and efficient way.

Fault analysis and diagnosis, fault tolerance. The design of fault tolerant systems is rather neglected. Some initial studies performed by students indicate that this subject is usually not approached in a systematic way.

\section{Acknowledgements}

Acknowledgements are due to our colleagues Sjaak Brinkkemper, Peter Moekotte, Patrick Ampting, Peter Rosendaal and George Steenbekke, and the students Udo Pijpker, Robbert Kramer, Carla Grosz Dias and Stef IJmker for their participation in the project.

\section{References}

[1] Booch, G., Object-oriented design with applications, Benjamin/Cummings, 1994.

[2] Gomaa, H., Software design methods for concurrent and real-time systems, Addison-Wesley, 1993.

[3] Hatley, D.J., and I.A. Pirbhai, Strategies for real-time system specification, Dorset House, 1987.

[4] Rumbaugh, J., M. Blaha, W. Premerlani, F. Eddy, and W.
Lorensen, Object-oriented modeling and design, PrenticeHall, 1991.

[5] Selic, B., G. Gullekson, and P.T. Ward, Real-time objectoriented modeling, John Wiley, 1994.

[6] Shlaer, S., and S.J. Mellor, Object lifecycles, modeling the world in states, Yourdon Press, Prentice-Hall, 1992.

[7] Shumate, K., and M. Keller, Software specification and design, a disciplined approach for real-time systems, John Wiley, 1992.

[8] Ward, P.T. \& S.J. Mellor, Structured Development Techniques for Real-Time Systems, Prentice-Hall, 1985.

[9] Young, S.J., Real time languages: design and development, Ellis Horwood, 1982.

[10] Weg, R.L.W. van de, Analysis and design of information systems based on an object-oriented framework, Ph.D. thesis, University of Twente, Enschede, 1995.

[11] Harel, D., et al., STATEMATE: A working environment for the development of complex reactive systems, IEEE Transactions on Software Engineering, Vol. 16, Nr. 4, April 1990, p. 403 - 414.

[12] Elmasri, R., and S.B. Navathe, Fundamentals of database systems, Benjamin/Cummings, 1994.

[13] Skvarcius, R., and W.B. Robinson, Discrete Mathematics with Computer Science Applications, Benjamin/Cummings, 1986.

[14] Beek, D.A. van, Exception handling in control systems, Ph.D. thesis, Eindhoven University of Technology, 1993.

[15] Levi, S.-T., and A.K. Agrawala, Fault tolerant system design, McGraw-Hill, 1994.

[16] ObjectMaker Manuals, Mark V Systems Limited, 16400 Ventura Boulevard, Encino, CA, USA. 